\title{
Capacitação em Ciência de Animais de Laboratório
}

\section{Training Course on Laboratory Animal Science}

\section{Resumo}

A Ciência de Animais de Laboratório envolve uma abordagem multidisciplinar com conhecimento do modelo animal e suas necessidades etológicas espécie-específicas, refinamento de experimentos que melhoram o bem-estar animal com reconhecimento e tratamento da dor, anestesia e eutanásia, bem como delineamento experimental e análise estatística dos resultados experimentais. Para atender à essas necessidades, pessoas que realizam, participam, supervisionam procedimentos experimentais em animais, ou cuidam de animais de laboratório, devem receber treinamento, com programas voltados à implementação dos 3Rs e promoção do bem-estar dos animais de laboratório, garantindo assim a qualidade e reprodutibilidade de experimentos com benefícios éticos, científicos e econômicos. Nos países da Europa e nos Estados Unidos, e requerido mais recentemente pelo Conselho Nacional de Controle de Experimentação Animal (CONCEA) no Brasil, um veterinário com experiência em medicina de animais de laboratório precisa fazer parte da equipe do Biotério. Este veterinário tem um papel fundamental na orientação de profissionais e pesquisadores em questões relacionadas ao bem-estar e cuidado com animais. Diante desta recomendação, fica clara a importância da formação de graduação e de médicos-veterinários para atuar na área de Animais de Laboratório, e poder desempenhar seu papel nos biotérios visando melhorar o cuidado e apoiar estudos experimentais que envolvem esses animais.

\section{Abstract}

Laboratory Animal Science requires a multidisciplinary approach. Professionals working with laboratory animals should have the knowledge on how to choose the right animal model and its speciesspecific ethological needs, how to choose the best anesthesia, analgesia and euthanasia protocol, how to refine the care procedures including recognition and treatment of pain in order to improve the animals welfare, as well as how to design an experimental and to conduct statistical analysis of the experimental data. To meet these needs, professionals who perform, take part, supervise experimental procedures on animals, or take care of laboratory animals, should receive training, with programs aimed to implement the $3 R s$ and to promote the welfare of laboratory animals, and therefore guarantee the quality and reproducibility of experiments with ethical, scientific and economic benefits. Veterinarians with experience in laboratory animal medicine are required to be part of the Animal Facility staff in Europe and in the United States, and most recently, in Brazil, established by the National Council for the Control of Animal Experimentation (Concea). Veterinarians working with laboratory animals have a key role in guiding other professionals and researchers regarding issues related to animal welfare and care. Since it is necessary to follow the Concea recommendation, it is visible the importance to have the veterinary undergraduate students and veterinarians properly trained in order to provide the best quality of life for the animals used in research and consequently having relevant, translatable scientific data and the most beneficial use of these animals. 
Ana Tada Fonseca Brasil Antiorio*1,2,5

Luciana Cintra ${ }^{1,3,5}$

Márcia Carolina Millán Olivato 4,5

Mauricio de Rosa Trotta ${ }^{4,5}$

Rosália Regina De Luca5

Dennis Albert Zanatto ${ }^{1}$

Claudia Madalena Cabrera Mori ${ }^{1,5}$
Av. Prof. Dr. Orlando Marques de Paiva, 87, Cidade Universitária

São Paulo/SP, Brasil

CEP: 05508-270

凶ana.tbrasil@gmail.com

\section{Palavras-chave}

Animais de laboratório. Responsabilidade

técnica. Legislação. Ética. Ensino.

\section{Keywords}

Laboratory animals. Technical responsibility.

Legislation. Ethics. Teaching.

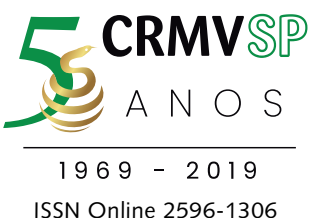

ISSN Online 2596-1306

\footnotetext{
ras
} 
A escolha do modelo animal mais apropriado, o delineamento experimental acurado, a análise estatística dos dados, o reconhecimento das necessidades etológicas espécie-específicas, o reconhecimento e tratamento da dor, a anestesia e a eutanásia são todas as etapas de grande importância para a realização de experimentos bem-sucedidos com animais. Todas as pessoas que realizam, participam, ou supervisionam os procedimentos, bem como aqueles que cuidam desses animais, devem ter recebido treinamento e formação adequados (FORNI, 2007). Nesse sentido, programas de treinamento voltados à Ciência de Animais de Laboratório devem promover a prática dos 3Rs e proporcionar bem-estar aos animais de laboratório, bem como garantir a qualidade e reprodutibilidade da ciência com benefícios éticos, científicos e econômicos.

Segundo a legislação vigente na Europa, as instituições que criam, fornecem ou utilizam animais devem ter como membro da equipe um médico-veterinário com experiência em medicina de animais de laboratório, encarregado de orientar questões relacionadas ao bem-estar e cuidados com os animais (POIRIER et al., 2015). O papel e as responsabilidades do médico-veterinário incluem a elaboração, implantação e revisão contínua de um programa adequado de cuidados veterinários em instituições que criam ou utilizam animais para fins científicos ou didáticos (Quadro 1).

Quadro 1 - Elementos que compõe o programa de cuidados veterinários em instalações que mantém animais para uso em pesquisa e ensino.

ATIVIDADES

Transporte de animais

Manejo e cuidados

Avaliação do bem-estar

Controle e prevenção de doenças

Uso de medicamentos

Identificação e controle da dor, sofrimento e estresse

Modelos animais

Intervenções cirúrgicas e não cirúrgicas

Anestesia e analgesia

Eutanásia

$3 R s$

Visitas de rotina
DETALHAMENTO

Recomendações e cuidados veterinários em relação ao transporte, exportação, importação ou alojamento em um novo biotério.

Recomendações em relação ao manejo, incluindo nutrição apropriada e programas de enriquecimento ambiental.

Aconselhamento sobre métodos para avaliação do bem-estar. Supervisão pelo veterinário e/ou exames físicos para avaliação do bem-estar antes da reutilização, na transferência para um novo alojamento, liberação para a natureza e ao término dos procedimentos experimentais.

Estabelecimento de um programa de vigilância sanitária, prevenção, detecção, tratamento e controle de doenças transmissíveis, incluindo zoonoses. Atendimento clínico. Aconselhamento em planos de emergência caso ocorra o surto de uma doença.

Aconselhamento sobre a permissão, armazenamento e uso de fármacos, incluindo medicamentos controlados.

Identificação e manejo de eventos adversos que impactem na saúde e bem-estar dos animais quando associados ao protocolo experimental ou não.

Aconselhamento em relação à escolha das espécies animais e linhagens mais adequadas para a pesquisa, incluindo Animais Geneticamente Modificados (AGM). Aconselhamento sobre os modelos animais e delineamento experimental.

Fornecer recomendações técnicas em procedimentos experimentais que envolvam intervenções cirúrgicas ou não.

Fornecer orientação e protocolos para anestesia, analgesia e cuidados pré e pós-operatórios adequados aos procedimentos experimentais.

Fornecer orientação e protocolos referentes às práticas de eutanásia.

Aconselhamento e implantação do princípio dos 3 Rs em relação a qualquer aspecto sobre o cuidado e uso de animais.

Realizar visitas com frequência adequada para monitorar a saúde e o bem-estar da colônia e demais procedimentos que possam afetar a saúde e bem-estar dos animais.

Fonte: Adaptado de Poirier et al. (2015).

\section{Histórico e legislação}

O primeiro colégio de Medicina de Animais de Laboratório foi fundado nos Estados Unidos da América em 1957, com a premissa de incentivar o ensino, treinamento e pesquisa em Ciência de Animais de Laboratório, promovendo a formação de médicos-veterinários especialistas em cuidados e sanidade dos animais de laboratório, além do reconhecimento desses profissionais por exames de certificação. Em 1961, esse colégio passou a se chamar American College of Laboratory Animal Medicine $\left(\mathrm{ACLAM}^{(3)}\right)$ e atualmente é responsável pela certificação de médicos-veterinários especialistas em Animais de Laboratório e por programas educacionais em toda a América do Norte, incluindo o Canadá (CLARK, 2007). 
Em 2005, os Colégios de Medicina de Animais de Laboratório existentes na América do Norte, Europa, Japão e Coreia do Sul uniram forças para formar o International Association of Colleges of Laboratory Animal Medicine $\left(\right.$ IACLAM $\left.^{(4)}\right)$. A missão do Iaclam abrange quatro grandes áreas: a) certificação de médicos-veterinários especialistas em Animais de Laboratório; b) formação e treinamento de médicos-veterinários, incluindo países em desenvolvimento como na América Latina, China e Índia; c) disseminação de conhecimento relevante à área; e d) colaboração em pesquisas, promovendo a implantação dos 3Rs e, principalmente, no que se refere ao papel do médico-veterinário no refinamento (CLARK, 2007). Além disso, o Iaclam dá suporte às iniciativas de outras organizações, como o International Committee on Laboratory Animal Science $\left(\operatorname{ICLAS}^{(5)}\right.$ ) e o Institute for Laboratory Animal Research (ILAR $\left.{ }^{(6)}\right)$, visando a harmonização dos conhecimentos e padronização internacional da Ciência de Animais de Laboratório.

$\mathrm{Na}$ Europa, a Federation of European Laboratory Animal Science Associations (FELASA) oferece treinamento para quatro categorias distintas, sendo elas: Categoria A - pessoas responsáveis pelos cuidados básicos com animais de experimentação; Categoria B - alunos e pesquisadores que executam experimentos com animais; Categoria $\mathbf{C}$ - pesquisadores responsáveis por chefiar grupos de pesquisas envolvendo animais de experimentação; e Categoria D - médicos-veterinários especialistas em Animais de Laboratório (GUILLEN, 2012).

OColégio Brasileiro de Experimentação Animal (COBEA) foi fundado em 1983 pelo Prof. Dr. Fernando Sogorb Sanchis com o objetivo de garantir o bem-estar e o uso racional dos animais de laboratório, capacitar profissionais e propor uma legislação específica. Em 2008, o COBEA passou a se chamar Sociedade Brasileira de Ciências em Animais de Laboratório (SBCAL), cujo caráter multidisciplinar atua na formação de profissionais nas diferentes áreas da Ciência em Animais de Laboratório, resumidas no Quadro 2.

A literatura nacional que aborda os cuidados e manejo das diferentes espécies de animais de laboratório tem sido ampliada, incluindo as publicações de Andrade, Pinto e Oliveira (2002), Lapchik, Mattaraia e Ko (2017), o Manual de Cuidados e Procedimentos com Animais de Laboratório do Biotério de Produção e Experimentação da Faculdade de Ciências Farmacêuticas e Instituto de Química da Universidade de São Paulo (FCF-IQ/USP) no formato de e-book (NEVES; MANCINI FILHO; MENEZES, 2013) e a Revista da Sociedade Brasileira de Ciências em Animais de Laboratório (RESBCAL).

\footnotetext{
3 Disponível em: http://www.aclam.org. Acesso em: 18 set. 2019.

4 Disponível em: http://www.iaclam.org. Acesso em: 18 set. 2019.

5 Disponível em: http://iclas.org/. Acesso em: 18 set. 2019.

6 Disponível em: http://dels.nas.edu/. Acesso em: 18 set. 2019.
}

Quadro 2 - Áreas de conhecimento em Ciência de Animais de Laboratório.

I - EDUCAÇÃO, BIOÉTICA, LEGISLAÇÃO E BIOSSEGURANÇA EM ANIMAIS DE LABORATÓRIO:

- Educação e formação de recursos humanos para criação e uso de animais de laboratório

- Ética e avaliação de projetos de pesquisa que envolvem animais de laboratório;

- Legislação e controle da produção e da experimentação animal;

- Medicina Veterinária em animais de laboratório;

- Biossegurança em biotérios de criação e experimentação.

II - BEM-ESTAR E COMPORTAMENTO ANIMAL:

- Bem-estar de animais de laboratório (reconhecimento e redução de estresse desconforto e dor, enriquecimento ambiental);

- Comportamento Animal.

III - PRODUÇÃO DE ANIMAIS DE LABORATÓRIO:

- Nutrição;

- Manejo;

- Reprodução de linhagens heterogênicas e isogênicas;

- Produção de novos modelos animais (transgênicos e geneticamente modificados);

- Controle de qualidade de animais de laboratório (sanitário, genético e ambiental);

- Criopreservação de gametas e embriões de animais de laboratório.

IV - MANIPULAÇÃO E EXPERIMENTAÇÃO EM ANIMAIS DE LABORATÓRIO:

- Métodos alternativos ao uso de animais de laboratório;

- Refinamento dos procedimentos utilizados em animais de laboratório;

- Inovações tecnológicas no uso de animais de laboratório;

- Fatores que afetam os resultados de experimentos científicos;

- Anestesia, analgesia e eutanásia.

V - EDIFICAÇÃO E GERENCIAMENTO DE BIOTÉRIOS:

- Reforma e construção de biotérios;

- Climatização de biotérios de criação e experimentação;

- Barreiras sanitárias: equipamentos e manutenção preventiva e corretiva;

- Novas tecnologias na manutenção de animais de laboratório;

- Controle reprodutivo e distribuição de animais.

Fonte: SBCAL (HISTÓRICO..., 2013)

No Brasil, a Lei 11.794 , de 8 de outubro de 2008, regulamentada pelo Decreto 6.899, de 15 de julho de 2009, conhecida como Lei Arouca, dispõe sobre procedimentos para o uso de animais para fins científicos e didáticos (BRASIL, 2008; BRASIL, 2009). A lei abrange vertebrados utilizados na experimentação científica, limitando o uso dos animais para atividades didáticas e científicas em instituições de ensino superior e ensino técnico na área biomédica. Ainda, determina a criação do Conselho Nacional de Controle da Experimentação Animal (CONCEA) no Ministério de Ciência e Tecnologia, a obrigatoriedade da instalação das Comissões de Ética no Uso de Animais (CEUA) e o registro e licenciamento da Instituição no Cadastro das Instituições de Uso Científico 
de Animais (CIUCA). Em 10 de julho de 2012, o CONCEA publicou a Resolução Normativa número 6 que instituiu a figura do Responsável Técnico pelos biotérios conforme redação do artigo 9, parágrafo II: [...]” o Responsável Técnico pelos Biotérios deverá ter o título de médico- veterinário com registro ativo no Conselho Regional de Medicina Veterinária da Unidade Federativa em que o estabelecimento esteja localizado e assistir aos animais em ações voltadas para o bem-estar e cuidados veterinários." (BRASIL, 2012, p. 13). Mais recentemente, em 20 de junho de 2018, foi publicada a Resolução Normativa número 39, que define a capacitação da equipe envolvida em procedimentos experimentais invasivos em animais de laboratório, incluindo o papel do médico-veterinário em procedimentos cirúrgicos com animais (BRASIL, 2018a).

A importância da capacitação profissional também é destacada nas normas do Conselho Federal de Medicina Veterinária (CFMV). Porém, um dos documentos mais relevantes que baliza a conduta do profissional é o Código de Ética do médico-veterinário. O texto diz que: "Art. $6^{\circ}$ São deveres do médico veterinário: I - aprimorar continuamente seus conhecimentos e usar o melhor do progresso científico em benefício dos animais, do homem e do meio-ambiente; [...]". (CFMV, 2017a, p. 108). Em 2017, o CFMV publicou a Resolução ${ }^{\circ} 1.178$, que dispõe especificamente sobre a responsabilidade técnica em estabelecimentos que criem ou utilizem animais em atividades de pesquisa ou ensino. $\mathrm{O}$ texto traz o seguinte trecho:

Art. $2^{\circ} \mathrm{O}$ responsável técnico deve: $I$ - possuir conhecimento e treinamento específico em Medicina Veterinária, na área de ciências de animais de laboratório, em procedimentos clínicos de rotina, experimentais, de emergência, patologia, Medicina Veterinária preventiva com destaque para biossegurança, saúde pública, zoonoses e para o bem-estar animal; [...]. (CFMV, 2017b, p. 131)

O Conselho Regional de Medicina Veterinária do Estado de São Paulo (CRMV-SP) também aborda a importância e necessidade de capacitação em um capítulo exclusivo do Manual de Responsabilidade Técnica e Legislação, cuja versão atualizada em 2019 destaca que o médico-veterinário, especialmente aquele que irá assumir responsabilidade técnica, deve se manter treinado e atualizado, o que pode ser reconhecido no trecho transcrito a seguir:

A responsabilidade técnica deve ser entendida como o processo que materializa conceitos, sendo o RT a figura central que responde ética, legal e tecnicamente pelos atos profissionais, devendo ter capacitação para planejar, orientar e coordenar processos e cadeias de produção, ocupando posições de interação entre as instituições públicas de fiscalização (Ministérios, Secretarias Estaduais e Municipais), entidades de fiscalização e prevenção da saúde humana (Anvisa, Nasf, CCZ), entidades de proteção ao consumidor (Procon, $M P)$ e o CRMV. (CRMV-SP, 2019, p. 25-26, grifo nosso).

[...] É de responsabilidade do profissional e recomenda-se que, além da sua formação na graduação universitária, ele busque e adquira treinamento específico na sua área de atuação, mantendo-se sempre atualizado e cumprindo as normas e resoluções do Sistema CFMV/CRMVs. Quando da ART (Anotação de Responsabilidade Técnica), recomenda-se comprovar a capacitação do profissional por meio da apresentação de certificados de participação em cursos, congressos, seminários e outros, referentes à área de atuação nos últimos 24 meses. (CRMV-SP, 2019, p. 31, grifo nosso)

Em outro trecho desse mesmo documento, no qual trata especificamente dos Requisitos e atribuições gerais do Responsáveis Técnicos (RT) em biotérios, destaca-se que "O RT, quando no exercício de suas funções, em biotérios, deve: [...] ter experiência na área e realizar treinamento contínuo [...]". (CRMV-SP, 2019, p. 100).

Fica claro, portanto, que a capacitação profissional e, especialmente para aqueles profissionais que atuam com Responsabilidade Técnica em biotérios, faz parte do aprimoramento necessário para o exercício da profissão e é um dever que não pode ser negligenciado.

Formação do médico-veterinário em Animais de Laboratório

Considerando esses fatos, torna-se evidente a importância da formação do aluno de graduação em Medicina Veterinária na área de Animais de Laboratório; no entanto, poucas universidades no Brasil oferecem disciplinas curriculares. Dentre elas, estaca-se a Universidade de São Paulo (USP) nos cursos de Medicina Veterinária da Faculdade de Medicina Veterinária e Zootecnia (FMVZ) e Faculdade de Zootecnia e Engenharia de Alimentos (FZEA), a Universidade Federal Fluminense (UFF) e Universidade Federal do Rio Grande do Sul (UFRGS). Na FMVZ/USP, a disciplina Criação de Animais de Laboratório foi implantada pelo Departamento de Patologia no ano de 1977 e, atualmente, a instituição oferece duas disciplinas no curso de graduação: 1- Criação de Animais de Laboratório, disciplina obrigatória com carga horária de 30 horas e 2- Princípios Éticos e Legais no Uso de Animais no Ensino e Pesquisa, disciplina eletiva com carga horária de 30 horas. O conteúdo programático abrange as principais áreas de atuação do médico-veterinário na Ciência de Animais de Laboratório. As disciplinas têm o objetivo de capacitar 
o corpo discente nas atividades de criação, manutenção e procedimentos experimentais que utilizam animais de laboratório, visando o bem-estar animal e atendendo a legislação vigente, assim como, no planejamento físico, biossegurança e procedimentos operacionais em biotérios.

Seguindo uma tendência internacional, o uso de animais no ensino de graduação tem sido progressivamente substituído por alternativas, como programas de computador, manequins e simuladores, cadáveres de animais obtidos de maneira ética e atividades relacionadas ao atendimento de casos clínicos de rotina. A combinação de tais alternativas pode atender melhor os objetivos de ensino, reduzir custos e evitar o impacto pedagógico e social negativo da experimentação animal (MARTINSEN; JUKES, 2005). Ademais, de acordo com a resolução normativa de número 38 do CONCEA, de 17 de abril de 2018, artigo primeiro:

Fica proibido o uso de animais em atividades didáticas demonstrativas e observacionais que não objetivem desenvolver habilidades psicomotoras e competências dos discentes envolvidos. Eartigo segundo: [...] as atividades didáticas que utilizem animais referenciadas no art. $1^{\circ}$ deverão ser integralmente substituídas por vídeos, modelos computacionais, ou outros recursos providos de conteúdo e de qualidade suficientes para manter ou para aprimorar as condições de aprendizado. (BRASIL, 2018b, p. 16)

Corroborando com esses fatos, cumpre ser destacada a necessidade do desenvolvimento de novas estratégias para o ensino de graduação do curso de Veterinária, voltadas para a disciplina Criação de Animais de Laboratório, oferecida pela FMVZ/USP. Nesse sentido, alguns projetos encontram-se em desenvolvimento no laboratório coordenado pela Profa. Dra. Claudia Mori. Em destaque há o projeto "Métodos substitutivos ao uso de animais vivos no ensino de graduação em Medicina Veterinária: procedimentos em roedores de laboratório" que atualmente envolve alunos de graduação e de pós-graduação, além de outros profissionais de áreas multidisciplinares. Para a execução desse projeto foram filmados e fotografados os procedimentos de manipulação e contenção de ratos de laboratório e as principais vias de acesso para administração de substâncias e coleta de sangue, com descrição detalhada de cada procedimento. $\mathrm{O}$ material didático editado foi inserido na plataforma do Laboratório Integrado de Química e Bioquímica (LABIQ) no portal "Boas Práticas em Experimentação Animal” (BPEA) (Figura 1).
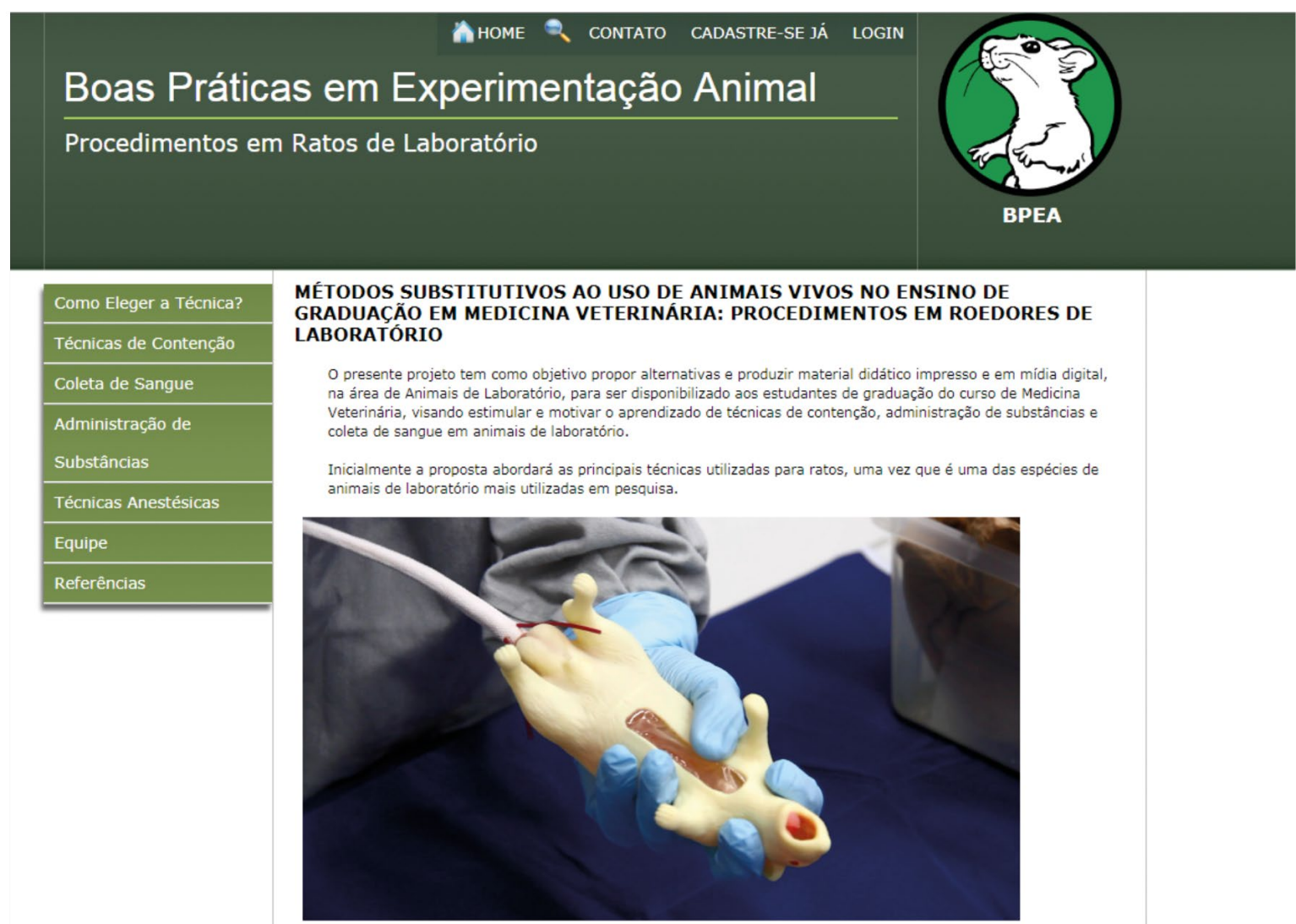

Figura 1 - Screenshot da página principal do portal "Boas Práticas em Experimentação Animal: Procedimentos em Ratos de Laboratório". Fonte: Zanatto et al. (2019). 
No programa de pós-graduação em Patologia Experimental e Comparada, a Profa. Dra. Claudia Mori orienta alunos de mestrado e doutorado em diferentes áreas da Ciência de Animais de Laboratório, como anestesia e analgesia, caracterização de novos modelos animais e métodos alternativos voltados ao ensino.

Em 2018 teve início o curso de educação a distância da Rede Nacional de Biotérios de Produção de Animais para Fins Científicos, Didáticos e Tecnológicos (REBIOTÉRIO) do Conselho Nacional de Desenvolvimento Científico e Tecnológico (CNPq) sobre "Capacitação no uso e manejo de animais de laboratório" chamada pública MCTIC/CNPq $n^{\circ}$ 22/2017 (CNPQ, 2017). O objetivo do curso é difundir e atualizar conceitos na área para capacitação de profissionais, tendo como público-alvo usuários que pretendam trabalhar ou continuar suas atividades em biotérios de produção, manutenção e experimentação.

\section{Considerações finais}

A Ciência de Animais de Laboratório agrega o conhecimento de profissionais de diferentes áreas, dentre elas do médico-veterinário, que atua na assistência médica aos animais utilizados em experimentação garantindo a sanidade e bem-estar deles.

Desta forma, destaca-se a importância de implementar a disciplina de Animais de Laboratório nos cursos de graduação em Medicina Veterinária com o objetivo capacitar os futuros profissionais para atuarem como Responsáveis Técnicos na gestão de produção e manutenção dos diferentes modelos animais em biotérios, bem como no seu uso ético em protocolos de ensino ou pesquisa. (:)

\section{Referências}

ANDRADE, A.; PINTO, S. C.; OLIVEIRA, R. S. (org.). Animais de laboratório: criação e experimentação. Rio de Janeiro: Fiocruz, 2002.

BALLS, M. Replacement of animal procedures: alternatives in research, education and testing. Laboratory Animals, Thousand Oaks, v. 28, n. 3, p. 193-211, 1994.

BRASIL. Lei $\mathrm{n}^{\circ} 11.794$, de 8 de outubro de 2008. Regulamenta o inciso VII do $\S 1^{\circ}$ do art. 225 da Constituição Federal, estabelecendo procedimentos para o uso científico de animais; revoga a Lei $n^{\circ}$ 6.638 , de 8 de maio de 1979; e dá outras providências. Diário Oficial da União: seção 1, Brasília, DF, p. 1, 9 out. 2008.

BRASIL. Decreto n 6.899, de 15 de julho de 2009. Dispõe sobre a composição do Conselho Nacional de Controle de Experimentação Animal - CONCEA, estabelece as normas para o seu funcionamento e de sua Secretaria-Executiva, cria o Cadastro das Instituições de Uso Científico de Animais - CIUCA, mediante a regulamentação da Lei $n^{\circ}$ 11.794, de 8 de outubro de 2008, que dispõe sobre procedimentos para o uso científico de animais, e dá outras providências. Diário Oficial da União: seção 1, Brasília, DF, p. 2, 16 jul. 2009.
BRASIL. Ministério da Ciência, Tecnologia e Inovação. Conselho Nacional de Controle de Experimentação Animal. Resolução Normativa $n^{\circ}$ 06, de 10 de julho de 2012. Diário Oficial da União: seção 1, Brasília, DF, p. 13, 11 jul. 2012.

BRASIL. Ministério da Ciência, Tecnologia e Inovação. Conselho Nacional de Controle de Experimentação Animal. Resolução Normativa $n^{\circ} 30$, de 2 de fevereiro de 2016. Diário Oficial da União: seção 1, Brasília, DF, p. 3, 3 fev. 2016.

BRASIL. Ministério da Ciência, Tecnologia e Inovação. Conselho Nacional de Controle de Experimentação Animal. Resolução Normativa $n^{\circ} 39$, de 20 de junho de 2018a. Diário Oficial da União: seção 1, Brasília, DF, p. 7, 25 jun. 2018a.

BRASIL. Ministério da Ciência, Tecnologia e Inovação. Conselho Nacional de Controle de Experimentação Animal. Resolução Normativa $n^{\circ} 38$, de 17 de abril de 2018b. Diário Oficial da União: seção 1, Brasília, DF, p. 16, 19 abr. 2018b.

CFMV - CONSELHO FEDERAL DE MEDICINA VETERINÁRIA. Resolução $n^{\circ} 1.138$, de 16 de dezembro de 2016. Aprova o código de Ética do Médico-veterinário. Diário Oficial da União: seção 1, Brasília, DF, p. 108, 25 jan. 2017a.

CFMV - CONSELHO FEDERAL DE MEDICINA VETERINÁRIA. Resolução $n^{\circ} 1.178$, de 17 de outubro de 2017. Dispõe sobre a responsabilidade técnica em estabelecimentos que criem ou utilizem animais em atividades de pesquisa ou ensino. Diário Oficial da União: seção 1, Brasília, DF, p. 131, 27 out. 2017b.

CLARK, J. M. A global vision for laboratory animal medicine. AATEX, Tokyo, v. 14, special issue, p. 735-737, 2007.

CNPQ - CONSELHO NACIONAL DE DESENVOLVIMENTO CIENTÍFICO E TECNOLÓGICO. Chamada pública n 22/2017: curso a distância para capacitação no uso e manejo de animais de laboratório. Brasília, DF: CNPq, 22 ago. 2017. Disponível em: https://bit.ly/2klB9uG. Acesso em: 14 jun. 2019.

CRMV-SP - CONSELHO REGIONAL DE MEDICINA VETERINÁRIA DO ESTADO DE SÃO PAULO. Manual de responsabilidade técnica e legislação. 4 ed. rev. São Paulo: CRMV-SP, 2019.

FORNI, M. Laboratory animal science: a resource to improve the quality of science. Veterinary Research Communication, Dordrecht, v. 31, s. 1, p. 43-47, 2007.

GUILLEN, J. FELASA guidelines and recommendations. Journal of the American Association for Laboratory Animal Science, Memphis, v. 51, n. 3, p. 311-321, 2012.

HISTÓRICO. Sbcal/Cobea, São Paulo, 28 set. 2013. Disponível em: https://bit.ly/2IUOXwS. Acesso em: 4 jun. 2019.

LAPCHIK, V. B. V.; MATTARAIA, V. G. M.; KO, G. M. Cuidados e manejo de animais de laboratório. 2. ed. São Paulo: Atheneu, 2017.

MARTINSEN, S.; JUKES, N. Towards a humane veterinary education. Journal of Veterinary Medical Education, Toronto, v. 32, n. 4, p. 454-460, 2005. 
NEVES, S. M. P.; MANCINI FILHO, J.; MENEZES, E. W. Manual de cuidados e procedimentos com animais de laboratório do biotério de produção e experimentação da FCF-IQ/USP. São Paulo: FCF-IQ/USP, 2013. Disponível em: https://bit.ly/2IXLeP7. Acesso em: 14 jun. 2019.

POIRIER, G. M. et al. ESLAV/ECLAM/LAVA/EVERI recommendations for the roles, responsibilities and training of the laboratory animal veterinarian and the designated veterinarian under Directive 2010/63/EU. Laboratory Animals, London, v. 49, n. 2, p. 89-99, 2015.

RUSSELL, W. M. S.; BURCH, R. L. The principles of humane experimental technique. London: Methuen, 1959.

ZANATTO, D. A. et al. Boas práticas em experimentação animal: procedimentos em ratos de laboratório, 2019. Bpea, São Paulo, 2019. Disponível em: http://bpeanimal.iq.usp.br. Acesso em: 14 jun. 2019. 